\title{
A depression relapse prevention programme improved adherence to medication and depressive symptoms but did not decrease relapses
}

Katon W,Rutter C, Ludman EJ, et al. A randomized trial of relapse prevention of depression in primary care. Arch Gen Psychiatry 2001 Mar;58:241-7.

QUESTION: In primary care patients with major recurrent depression or chronic depression, does a relapse prevention programme improve adherence to medication and decrease symptoms and relapses?

\section{Design}

Randomised (allocation concealed*), blinded (outcome assessors)*, controlled trial with 12 months of follow up.

\section{Setting}

4 primary care clinics in Puget Sound, Washington, USA.

\section{Patients}

386 patients who were $18-80$ years of age (mean age 46 y, $74 \%$ women); had a diagnosis of depression or anxiety, and a recent antidepressant prescription (no previous prescriptions in the past 120 days); and were considered to be at high risk of relapse $(<4 D S M-I V$ major depressive symptoms and a history of $\geqslant 3$ episodes of major depression or dysthymia, or 4 residual depressive symptoms but a mean 20 item Hopkins Symptom Checklist [SCL-20] score of $<1.0$ and a history of major depression or dysthymia). Exclusion criteria included a risk of alcoholism, pregnancy or lactation, currently seeing a psychiatrist, or recent use of lithium or antipsychotic drugs. Follow up was $84 \%$.

\section{Intervention}

Patients were allocated to a relapse prevention programme $(n=194)$ or usual care $(n=192)$. The intervention included a self study book and videotape; 2 visits with a depression specialist; 3 telephone visits at 1,4 , and 8.5 months; and 4 personalised feedback mailings at 2 , 6,10 , and 12 months. Usual care involved 2-4 visits with family physicians in the 4 primary care clinics during the first 6 months and an option to refer to the mental health services.

\section{Main outcome measures}

Adherence to antidepressant medication and depressive symptoms.

\section{Main results}

Patients who received the relapse prevention programme were more likely than usual care group patients to refill antidepressant prescriptions (adjusted odds ratio [OR] $1.91,95 \%$ CI 1.37 to $2.65, \mathrm{p}<0.001)$ and were more likely to receive adequate dosage of antidepressants (adjusted OR 2.08, CI 1.41 to 3.06, p <0.001). Patients who received the relapse prevention programme had fewer depressive symptoms. During the 1 year follow up, the SCL-20 scores were about 0.08 points lower than those of usual care group patients $(p=0.04)$. Prevention programme and usual care group patients did not differ for rates of relapse $(35.0 \% v$ $34.6 \%)$.

\section{Conclusions}

In primary care patients with major recurrent depression or chronic depression, a relapse prevention programme improved adherence to medication and decreased depressive symptoms. It did not decrease episodes of relapse.

*See glossary.

\section{COMMENTARY}

The study by Katon $e t$ al reinforces how elusive a depression disorder is. Many factors contribute to its successful treatment, and understanding and acknowledgement of the condition may be key features. This well done study showed increased compliance, but the bottom line is that this has no effect on depression relapse, which is disappointing and mainly caused by the compliance of the control group.

Several explanations can account for this. Firstly, the wide age range of the patients may capture different aetiologies and attitudes to the illness. In older patients, resistant depression may have a large cerebrovascular component, which will reduce response to antidepressants and increase the risk of relapse. Conversely, younger patients are often less tolerant of symptoms and report them earlier, and some have their depression treated but remain in environments that increase the risk of recurrence. Thus a heterogeneous patient sample exists in this study, and subgroup analysis of the data might be revealing.

Secondly, the outcome measures for depression do not often reflect what is happening in the brain itself, so the phenotype we measure is not that accurate. The outcome measures for this study were relatively simple, thus making judgements of them difficult. Having fewer depressive symptoms does not mean being disease free and the relation between response and relapse is still not clearly understood. The course of depression may be different during the disease than during a recovery phase, accounting for variance in the results-which may show fluctuation in a state of ill being rather than a treatment and relapse effect.

Thirdly, the control group in most mental health studies has a substantial treatment component, especially once the control group patients have read the study aims, information leaflet, and consent form, and been exposed to more attention than usual.

If none of the explanations above are true in this study, we must face the stark conclusion that our management of depression in the long term patient is completely unhelpful, which I doubt is the case. Once again, a new study shows the need for more work.

Roger Bullock, MA, MBBS, MRCPsych Victoria Hospital Swindon, UK

\section{website $e x t r a$ \\ Additional references appear on the Evidence-Based Mental Health website www.ebmentalhealth. com}

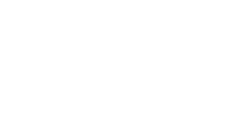

(1)

Source of funding: National Institute of Mental Health.

For correspondence: Dr W Katon, Department of Psychiatry, University of Washington School of Medicine, Box 35-6560, Seattle, WA 98195, USA. Fax +1 2062215414 